\title{
Carga de enfermedad por lesiones de causas externas en Bucaramanga, Colombia 2017
}

\author{
Burden of disease by external causes of injury \\ in Bucaramanga, Colombia 2017
}

\author{
Lina Fernanda Casadiegos-Patiño $^{1}{ }^{\text {D) }}$; Rafael Enrique Esquiaqui-Felipe ${ }^{1}{ }^{\mathbb{D}}$; Gloria Yadira Serrano-Diaz ${ }^{1}$
}

Forma de citar: Casadiegos Patiño LF, Esquiaqui Felilpe RE, Serrano Díaz GY. Carga de enfermedad por lesiones de causas externas en Bucaramanga, Colombia 2017. Salud UIS. 53: e21017. doi: https://doi.org/10.18273/saluduis.53.e:21017 @6 (1)

\section{Resumen}

Introducción: las lesiones por causa externa son uno de los principales problemas de salud pública en el mundo, y la metodología estandarizada de carga de enfermedad a través de los años de vida saludable perdidos (AVISAS) permite conocer el estado de salud poblacional y priorizar acciones. Objetivo: determinar la carga de enfermedad en términos de discapacidad y muerte como consecuencia de lesiones por causas externas en Bucaramanga, 2017. Diseño metodológico: estudio descriptivo de carga de la enfermedad. Se estimó los AVISAS producidos por LCE utilizando las bases de datos del Departamento Administrativo Nacional de Estadísticas, Sistema de Nacional de Vigilancia en Salud Pública y Registro Individual de la Prestación de Servicios de Salud, del año 2017, de la ciudad de Bucaramanga. Resultados: se estimó una carga global de enfermedad por LCE de 12,04 AVISAS por cada 1000 personas; 0,51 AVISAS atribuibles a discapacidad y 11,53 AVISAS, a mortalidad. Las agresiones y accidentes de tránsito (AT) son las dos principales LCE con mayor número de AVISAS. En la población de 5-59 años las agresiones y AT presentan AVISAS por mortalidad de mayor peso; los AT son la principal causa externa en la población de 60 a 79 años y la segunda en mayores de 80 años. Conclusiones: considerando que los AT fueron la causa externa con las más altas AVISAS atribuidas a mortalidad prematura, se recomienda implementar o intensificar estrategias de alto impacto que contribuyan a disminuir los AT.

Palabras clave: Carga de la Enfermedad; Mortalidad Prematura; Años Potenciales de Vida Perdidos. Causas Externas; Accidentes de Tránsito; Prioridades en Salud.

\begin{abstract}
Introduction: Injuries due to external causes are one of the main worldwide public health problems. The standardized methodology to evaluate burden diseases through the disability-adjusted life years (DALYs) allows us to know the health condition in a population and prioritize actions. Objective: To determine the burden of disease in terms of disability and death as consequence of injuries due to external causes in Bucaramanga, 2017. Methodology: descriptive study was conducted. The DALYs produced by injuries due to external causes were estimated using

1. Universidad de Santander. Bucaramanga, Colombia.

Correspondencia: Lina Fernanda Casadiegos Patiño. Dirección: Calle 90 a 17c-47. Bucaramanga. Santander. Colombia. Teléfono: +57 3164952410. Correo electrónico: linacasadiegosp@gmail.com
\end{abstract}


information from the National Administrative Department of Statistics, the National Public Health Surveillance System and the Individual Registry of Service Provision of Health databases from 2017 for the city of Bucaramanga, Colombia. Results: The global burden of diseases due to external causes was estimated at 12.04 DALYs per 1000 inhabitants; 0.51 DALYs were attributed to disability and 11.53 DALYs to mortality. Assaults and road traffic accident injuries (RTAI) were the main external causes with higher DALYs. In the population between 5 and 59 years old, assaults and RTAI had the highest DALYs attributed to mortality. RTAI were the first external cause of DALYSs in the population between 60 and 79 years old, and the second cause in the population older than 80 years. Conclusion: Considering that RTAI was the external cause with the highest DALYs attributed to premature mortality, it is recommended to implement or intensify high-impact strategies to reduce RTAI.

Keywords: Burden of Disease; Premature Mortality; Years of Potential Life Lost; External Causes; Traffic Accidents; Health Priorities.

\section{Introducción}

Las lesiones por causa externa (LCE) son aquellas definidas como el daño o lesión causado en una persona en forma intencional o no intencional, el cual puede originarse debido a un accidente, traumatismo, agresión, envenenamiento, etc., y se clasifica como lesión fatal o no fatal ${ }^{1}$. Son consideradas como uno de los principales problemas de salud pública en el mundo. En Colombia, las LCE van en aumento a expensas del trauma por accidente de tránsito y suicidio, generando una alta carga de enfermedad en el país ${ }^{2}$. Desde el año 2010, según la Organización Mundial de la Salud (OMS), se conoció que aproximadamente el 10\% del total de la mortalidad anual en el mundo (5,8 millones de personas) es por estas causas, y, además, por cada una de estas muertes existen miles de personas lesionadas, muchas de ellas con secuelas permanentes ${ }^{3}$.

La OMS refiere que anualmente 1,35 millones de personas en el mundo mueren a causa de lesiones por accidentes de tránsito, $\mathrm{y}$, además, que es la principal causa de muerte en la población entre los 5 y los 29 años. En la región de las Américas el $11 \%$ del total de las muertes son consecuencia de los accidentes de tránsito, lo que representa casi 155000 defunciones $^{4}$.

En Colombia para el año 2017, entre las diez primeras causas de mortalidad, que representan el $55,6 \%$ del total de las defunciones a nivel nacional, se encuentran dos de los cuatro subgrupos de las LCE (agresiones u homicidios y accidentes de tránsito). Entre las causas externas de defunción, los dos primeros lugares fueron ocupados por las agresiones (homicidios) con un 42,2\% (12 093 casos) y los accidentes de tránsito con un $25,2 \%(7224 \text { casos })^{5}$.
Las investigaciones en el país sobre carga de la enfermedad se han realizado durante los años 1994, 1995, 2005, 2010 y 2012, y han permitido una aproximación más local a la problemática ${ }^{6}$. En Bucaramanga, la carga global de enfermedad para 2016 fue estimada en 151 AVISAS (años de vida saludables potencialmente perdidos) por cada 1000 habitantes, de las cuales 59 fueron atribuidas a discapacidad y 92 a mortalidad. En cuanto al sexo y la edad, se encontró que, en la distribución de AVISAS de discapacidad y mortalidad en los hombres, los rangos etarios de 5-14 años y 15-29 años eran los grupos con mayor discapacidad, mientras que en las mujeres los grupos con AVISAS por discapacidad más altos se encontraban en los rangos 4-14 años y 29-44 años².

Según los datos consignados en la investigación anteriormente mencionada, en los hombres los accidentes de tránsito son la quinta causa según AVISAS en la ciudad (6517 AVISAS totales). Además, las AVISAS en discapacidad por cada 1000 habitantes fueron de 0,074 y las AVISAS en mortalidad por cada 1000 habitantes fueron de 6442. En el caso de las mujeres, los accidentes de tránsito no figuran entre las causas según AVISAS. Lo anterior permite determinar que los accidentes de tránsito son una problemática en la que están involucrados especialmente los hombres².

Finalmente, Montero et al. refieren que en la ciudad se ha dado una disminución importante de la carga de enfermedad relacionada con LCE, especialmente, en lo referente a mortalidad; panorama similar en el resto del país, pues a partir del año 2000 la reducción se ha situado en un $28 \%$ para los hombres y un $38 \%$ para las mujeres ${ }^{7}$. 
Sin embargo, a pesar del panorama anteriormente descrito, son escasos los estudios que miden el impacto de las lesiones por causas externas en la escala métrica AVISAS, los cuales permiten la estimación de la carga asociada a estos en Colombia, especialmente, en Bucaramanga. Asimismo, permiten tener más información para la toma de decisiones en términos de prevención y manejo de sus efectos en la sociedad.

En este sentido, es importante determinar los años de vida saludables potencialmente perdidos por muerte prematura y discapacidad, relacionados por las LCE en Bucaramanga, con el propósito de establecer el impacto de estas problemáticas en la calidad de vida, $\mathrm{y}$, así, poder priorizar y focalizar las intervenciones intersectoriales por edad, sexo y causas. Por tanto, el objetivo del presente estudio fue determinar la carga de enfermedad en términos de discapacidad y muerte como consecuencia de lesiones por causas externas en Bucaramanga, durante el año 2017.

\section{Metodología}

Se desarrolló un estudio descriptivo retrospectivo que permitió determinar la carga de enfermedad, teniendo como unidad de análisis los años de vida saludables potencialmente perdidos (AVISAS) ${ }^{8}$. Estos son una medida de salud que suma el tiempo de vida perdido como consecuencia de una muerte prematura (AVPM) y las pérdidas de salud referidas a los años vividos con una discapacidad (AVD), relacionados con los diagnósticos CIE-10 de lesiones por causas externas, presentados en la ciudad de Bucaramanga durante el año 2017. En la Figura 1 se relacionan las fuentes de información y los procedimientos relacionados con las AVISAS, AVPM y AVD. Además, se pueden observar las etapas previas de evaluación de la calidad del dato y los procesos de reclasificación de códigos basuras, signos y síntomas mal definidos.

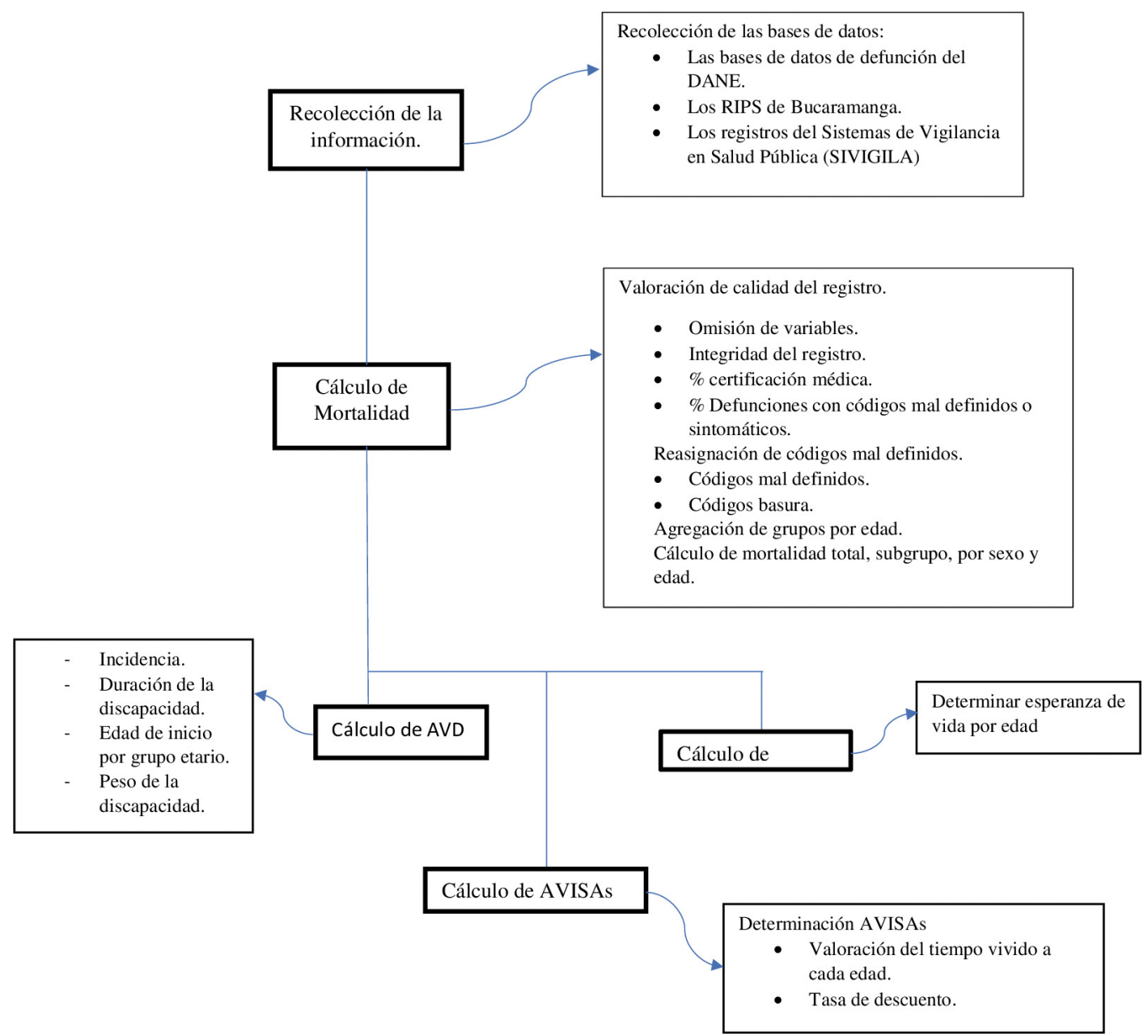

Figura 1. Flujograma carga de enfermedad por lesiones externas en Bucaramanga, 2017. 


\section{Estimación de la población}

Se utilizaron los registros del Sistema de Vigilancia en Salud Pública (SIVIGILA), los Registros Individuales de Prestación de Servicios (RIPS) y los registros de mortalidad relacionados con causas externas en la ciudad de Bucaramanga, del año 2017\%; se construyeron tablas de poblaciones por grupos de edades $(0-4,5-14$, $15-29,30-44,45-59,60-69,70-79$ y > 80 años).

\section{Estimación de la mortalidad}

Para el cálculo de la tasa de mortalidad por LCE, el numerador fue el número de muertes ocurridas en el año 2017 por los diagnósticos CIE-10 relacionados con LCE, según el reporte de defunciones del DANE $^{9}$ y el denominador de la población (total, por subgrupo, por edad y/o sexo, según corresponda) del mismo año, a partir del Censo 2005 (DANE), y que por convención corresponde a la población en la mitad del año. Las tasas se expresaron por cada habitante.

\section{Asignación de la discapacidad}

Se utilizaron las tablas de secuelas y discapacidad atribuible a cada enfermedad según los cálculos provistos por Murray \& López ${ }^{10}$.
Cálculo de los años de vida perdidos por muerte prematura (AVPM) y los años de vida perdidos por discapacidad (AVD)

Para el procesamiento de datos de estos dos indicadores se utilizaron la tabla de esperanza de vida japonesa, publicada por Murray ${ }^{11}$, ajustada con descuento del $3 \%$ y ponderada desigual por edades ( $B=0,04$ y $C=0,1658)$, y las salidas originarias de la utilización del Dismod (casos incidentes, duración y la edad de inicio); esto con el fin de garantizar una apropiada consistencia entre los datos ${ }^{10,12}$.

\section{Estimación de los años de vida (AVISAS)}

La estimación de los AVISAS por edad, sexo y LCE (grupo, subgrupo y tipo de víctima) se realizó mediante la siguiente ecuación: AVISAS $=$ AVPM + AVD.

\section{Resultados}

La carga global de enfermedad en la ciudad de Bucaramanga durante el año 2017 por causas externas se estimó en 12,04 AVISAS totales por cada 1000 habitantes, 0,51 AVISAS atribuible a discapacidad y 11,53 AVISAS de mortalidad por 1000 habitantes; las dos principales causas externas con mayor número de AVISAS son agresiones y accidentes de tránsito (véase Tabla 1).

Tabla 1. Clasificación de las causas externas según AVISAS totales (x 1000 personas) en ambos sexos y para todas las edades, Bucaramanga, carga de enfermedad, 2017.

\begin{tabular}{|c|c|c|c|c|}
\hline Orden & Causa & $\begin{array}{c}\text { AVISAs } \\
\text { Totales x 1000personas }\end{array}$ & $\begin{array}{c}\text { AVISAs } \\
\text { Discapacidad x } 1000 \text { personas }\end{array}$ & $\begin{array}{c}\text { AVISAs } \\
\text { Mortalidad x } 1000 \text { personas }\end{array}$ \\
\hline 1 & Agresiones & 4,493 & 0,009 & 4,484 \\
\hline 2 & Accidentes de tránsito & 3,52 & 0,037 & 3,483 \\
\hline 3 & $\begin{array}{l}\text { Otraslesiones no } \\
\text { intencionales (Residual) }\end{array}$ & 1,506 & - & 1,506 \\
\hline 4 & $\begin{array}{l}\text { Lesiones autoinfligidas } \\
\text { intencionalmente }\end{array}$ & 1,256 & 0,206 & 1,05 \\
\hline 5 & Caídas & 0,669 & 0,005 & 0,664 \\
\hline 6 & $\begin{array}{l}\text { Ahogamiento y sumersión } \\
\text { accidentales }\end{array}$ & 0,281 & 0,001 & 0,28 \\
\hline 7 & Envenenamiento accidental & 0,204 & 0,138 & 0,066 \\
\hline 8 & $\begin{array}{l}\text { Picadura de animal } \\
\text { venenoso }\end{array}$ & 0,109 & 0,109 & - \\
\hline \multirow[t]{2}{*}{9} & $\begin{array}{l}\text { Exposición al humo, fuego } \\
\text { yllamas }\end{array}$ & 0,005 & 0,005 & - \\
\hline & TOTAL & 12,042 & 0,51 & 11,533 \\
\hline
\end{tabular}

AVISAs=Años perdidos ajustados por discapacidad. 
En la Figura 2 se pueden observar los pesos relativos que tienen los AVPM y AVD en la carga global. La carga de enfermedad global se debe en un $95 \%$ a las muertes prematuras, mientras que solo el $4,2 \%$ se relacionan a discapacidad. Por sexo, la diferencia es ligeramente menor, pues para los hombres el $2 \%$ de los AVISAS totales corresponden a AVD, mientras que en las mujeres esta proporción asciende a $13 \%$.

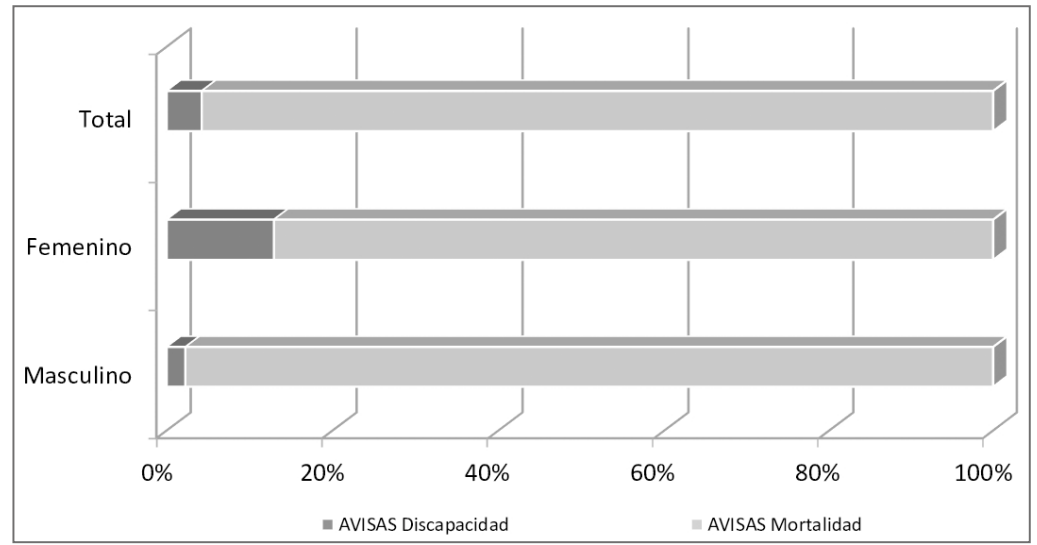

Figura 2. Pesos relativos de AVPM y AVD (\%) por sexo, carga de enfermedad. Bucaramanga, 2017.

La distribución de AVD y AVPM por grupos de edad y sexo presenta los perfiles que se muestran en las Figuras 3 y 4, para hombres y mujeres. Resultan ser diferentes los perfiles de distribución de la carga entre géneros, observándose que en los hombres los grupos de edad con AVPM más altos son los de 70-79 y mayores de 80 años, mientras que en las mujeres los grupos con AVPM más altos son los de 30-44 y 60-69 años. En cuanto a las AVD, existe una mayor similitud entre los dos géneros; se observa que en los hombres los grupos de edad más altos son los de 5-14 y 0-4 años, y en las mujeres son los de 5-14 y 15-29 años.

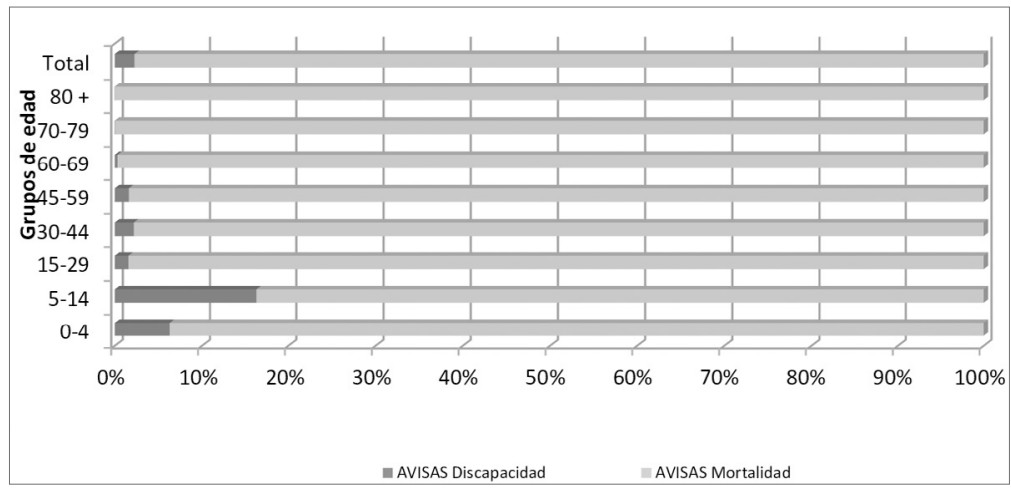

Figura 3. Pesos relativos AVD y AVPM (\%) en hombres por edad, carga de enfermedad. Bucaramanga, 2017.

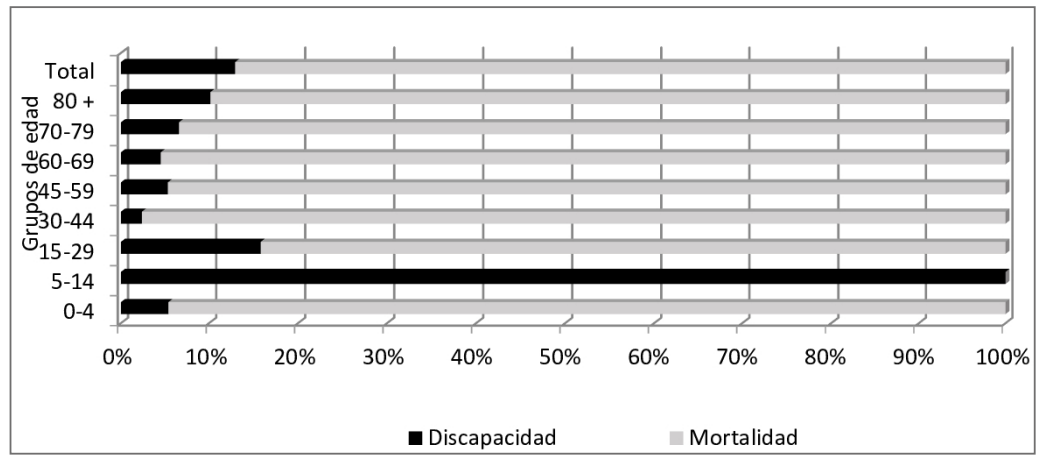

Figura 4. Pesos relativos AVD y AVPM (\%) en mujeres por edad, carga de enfermedad. Bucaramanga, 2017. 
Finalmente, como se observa en la Tabla 2, los accidentes de tránsito son una de las primeras causas externas que afecta tanto a hombres como a mujeres, esto dado en ambos grupos, principalmente, por las AVISAS por AVPM.

Tabla 2. Clasificación de las causas externas según AVISAS totales (x 1000 personas) y sexo, para todas las edades, Bucaramanga, carga de enfermedad, 2017.

\begin{tabular}{|c|c|c|c|c|c|c|c|c|}
\hline \multicolumn{5}{|c|}{ Hombres } & \multicolumn{4}{|c|}{ Mujeres } \\
\hline Orden & Causa & $\begin{array}{c}\text { AVISAs } \\
\text { Totales x } 1000 \\
\text { personas }\end{array}$ & $\begin{array}{c}\text { AVISAs } \\
\text { Discapacidad } x \\
1000 \text { personas }\end{array}$ & $\begin{array}{c}\text { AVISAs } \\
\text { Mortalidad x } \\
1000 \text { personas }\end{array}$ & Causa & $\begin{array}{c}\text { AVISAs } \\
\text { Totales } \mathrm{x} \\
1000 \text { personas }\end{array}$ & $\begin{array}{c}\text { AVISAs } \\
\text { Discapacidad } x \\
1000 \text { personas }\end{array}$ & $\begin{array}{c}\text { AVISAs } \\
\text { Mortalidad } x \\
1000 \text { personas }\end{array}$ \\
\hline 1 & Agresiones & 8,501 & 0,015 & 8,485 & $\begin{array}{l}\text { Accidentes de } \\
\text { tránsito }\end{array}$ & 1,201 & 0,022 & 1,179 \\
\hline 2 & $\begin{array}{l}\text { Accidentes de } \\
\text { tránsito }\end{array}$ & 6,017 & 0,053 & 5,964 & $\begin{array}{c}\text { Lesiones } \\
\text { autoinfligidas } \\
\text { intencionalmente }\end{array}$ & 0,924 & 0,273 & 0,651 \\
\hline 3 & $\begin{array}{l}\text { Otras lesionesno } \\
\text { intencionales } \\
\text { (Residual) }\end{array}$ & 2,517 & - & 2,517 & Agresiones & 0,772 & 0,003 & 0,769 \\
\hline 4 & $\begin{array}{c}\text { Lesiones } \\
\text { autoinfligidas } \\
\text { intencionalmente }\end{array}$ & 1,613 & 0,134 & 1,480 & $\begin{array}{l}\text { Otras lesiones no } \\
\text { intencionales } \\
\text { (Residual) }\end{array}$ & 0,567 & - & 0,567 \\
\hline 5 & Caídas & 1,030 & 0,004 & 1,026 & Caídas & 0,333 & 0,006 & 0,328 \\
\hline
\end{tabular}

\section{Discusión}

En Colombia se han realizado investigaciones de carga de enfermedad, algunas de las cuales no permiten comparar los resultados de este estudio por presentar modificaciones y/o ajustes en su metodología. Cabe resaltar que existen dos estudios principales que utilizan completamente la metodología de carga de enfermedad propuesta por Christopher Murray. Estos estudios fueron realizados en 1994 por el Ministerio de Salud y en el 2010 y 2012 por la Universidad Javeriana; sin embargo, presentaron diferentes estimaciones directas de AVISAS en mortalidad y discapacidad.

En función de los resultados encontrados en este estudio, la carga global de enfermedad en el municipio de Bucaramanga durante el año 2017 por causas externas se estimó en 12,04 AVISAS totales por cada 1000 habitantes, 0,51 AVISAS atribuibles a discapacidad por 1000 habitantes y 11,53 AVISAS de mortalidad por
1000 habitantes; las agresiones y accidentes de tránsito son las dos principales causas externas con mayor número de AVISAS.

Por su parte, los resultados de un estudio realizado en Bucaramanga en el año 2016 muestran que la carga global de enfermedad estimó 152 AVISAS por cada 1000 habitantes, de las cuales 59 fueron atribuidas a discapacidad y 92 a mortalidad ${ }^{2}$. Sin embargo, Estos datos no pueden ser comparables al no tener resultados de las AVISAS para el subgrupo III (LCE), las cuales están implícitas en la carga total calculada; no obstante, las agresiones y accidentes de tránsito se encuentran entre las 10 primeras causas de mayor carga de enfermedad en la población ${ }^{2}$.

Así mismo, Esquiaqui, en su estudio del año 2016, evidenció que las primeras causas externas de muerte prematura, con 4316 y 3749 AVISAS, fueron las agresiones y accidentes de tránsito, respectivamente; 
además, encontró que en los jóvenes (15-29 años), el $60 \%$ de los AVISAS totales se debe a discapacidad ${ }^{2}$. Inclusive en el 2017, con los resultados obtenidos en el presente estudio, se determinó que las agresiones y accidentes de tránsito continúan siendo las dos primeras causas externas que afectan tanto a hombres como a mujeres, debido principalmente a las AVISAS por AVPM. Al observar el grupo de 0-4 años, en los hombres se produjeron 8,36 AVISAS totales por cada 1000 personas; se identificaron las caídas como causa principal, seguidas del ahogamiento y los accidentes de tránsito.

Principalmente, en los hombres de 5-59 años, las agresiones y los accidentes de tránsito fueron el comportamiento de la carga de enfermedad por causas externas; no obstante, en los mayores de 80 años la mayor carga de enfermedad correspondió a las caídas.

En contraste, en una investigación realizada en Casanare se evidenció que la carga de enfermedad por notificación de LCE al SIVIGILA, expresada en los AVAD, fue de 2,5 años por 1000 habitantes, afectando principalmente a los hombres. Las intoxicaciones fueron la segunda causa de AVAD, resultando mayor en mujeres. En las lesiones por pólvora, los AVAD son aportados por hombres, mientras que las mujeres presentan más $\mathrm{AVAD}^{13}$ por violencia.

Según el informe de la OMS sobre el estado mundial de la seguridad vial del año 2018, se evidencia que las muertes anuales por accidentes de tránsito han alcanzado los 1,35 millones a nivel mundial $\mathrm{y}$, además, estos accidentes son la principal causa de muerte entre las personas de 5-29 años ${ }^{4}$. La mortalidad causada por los accidentes de tránsito es considerada como un problema de salud pública, el cual se ratifica de acuerdo con los resultados encontrados en el presente estudio.

Las dos principales causas externas que afectaron la carga de enfermedad en la población de 5-59 años en Bucaramanga durante el año 2017 fueron agresiones y accidentes de tránsito, encontrándose dentro de las AVISAS por mortalidad el mayor peso en la carga. Además, se evidencia que los accidentes de tránsito pasan a ser la principal causa externa en la población de 60-79 años y la segunda causa en mayores de 80 años; no obstante, en este último grupo las caídas son la primera causa externa que afecta la carga de enfermedad.

\section{Conclusiones}

El presente estudio de carga de enfermedad se focalizó en el grupo de causas externas para determinar los años de vida saludables potencialmente perdidos por muerte prematura y discapacidad en población de Bucaramanga, con el propósito de dar una importancia a este grupo de causas y, con ello, brindar criterios científicos y epidemiológicos válidos a los diferentes tomadores de decisiones con los cuales cuenta el municipio.

La carga de enfermedad aproxima a conocer mejor cuáles son las causas externas sobre las que se puede intervenir para mejorar la salud y prevenir la discapacidad de la población de Bucaramanga, esto a través de la medida de los AVISAS. Sin embargo, la falta de información de estudios a nivel nacional de LCE dificulta el análisis por regiones de la LCE.

En definitiva, tal como refiere la OMS, el precio que se está pagando por la movilidad es demasiado alto, especialmente, porque ya existen medidas eficaces que evitarían estas muertes. Entre ellas se encuentran las estrategias relacionadas con el comportamiento, especialmente, con la velocidad, el consumo de alcohol y la conducción, además de la creación de infraestructura vial más segura como, por ejemplo, los carriles exclusivos para los ciclistas y motociclistas ${ }^{4}$.

Teniendo en cuenta los hallazgos de la presente investigación, en el municipio de Bucaramanga se deben iniciar, impulsar o rediseñar estrategias de alto impacto para disminuir la AVISAS atribuibles a discapacidad y mortalidad por AT. Las agresiones y los accidentes viales son las principales causas externas con mayor número de AVISAS en el año 2017 en Bucaramanga, por lo tanto, estas estrategias deben estar orientadas a la población más afectada por los accidentes de tránsito que tienden a un aumento en la mortalidad, pasando de 6562 en el 2008 a 7224 en el 2017 (Departamento Administrativo Nacional de Estadística, 2018).

Para el municipio es importante estimar los años de vida perdidos por muerte prematura (AVPM), dado que con esta información se podrían generar proyectos costo-efectivos que disminuyan las lesiones por causas externas y, a su vez, disminuyan los años perdidos por estas causas. 
Por último, no se debe olvidar que existe otra población afectada, el adulto mayor, la cual debe ser incluida en planes de acompañamiento y prevención en caídas, realizando campañas de educación y manejo psicoafectivo y cansancio del rol cuidador con los familiares o acompañante permanente del adulto mayor.

Se recomiendan acciones intersectoriales entre las direcciones de tránsito, secretarias de salud, de desarrollo social e institutos municipales de cultura y turismo que enfoquen las acciones en los puntos críticos de accidentalidad y actividades de promoción y prevención a cuidadores de adultos mayores y salud mental en población adolescentes y adulto joven.

\section{Limitaciones}

Existe escasa información de las tasas de remisión, prevalencia y sobrevida de las LCE en el ámbito nacional que permita comparar estos insumos en la fase del modelamiento de los datos. Por otra parte, cabe resaltar que la metodología establecida para este tipo de estudio ha sido ampliamente cuestionada como sucede con la incorporación de la esperanza de vida japonesa, las tasas de descuentos y pesos de discapacidad. Sin embargo, han sido evaluadas a través de los análisis de sensibilidad en investigaciones como el estudio de "Carga de enfermedad Colombia" (2005) y en el estudio de "Carga de enfermedad por cáncer" (2005), encontrándose que no inciden en los resultados del orden de la priorización.

\section{Referencias}

1. Organización Panamericana de la Salud; Departamento de Epidemiología Hospital Escuela. Boletín vigilancia Epidemiológica de lesiones de causa externa. Segunda edicion, 2008. https:// www.paho.org/hon/index.php?option $=\mathrm{com}$ d o c man \& vi ew = down load \& c a te gory _ slug=desarrollo-humano-sostenible-y-estilosde-vida-sal\&alias=125-boletin-de-lvigilanciaepidemiologica-de-lesiones-de-causa-externa-2daedicion\&Itemid $=211$

2. Esquiaqui-Felipe R. Estudio de Carga de Enfermedad. Bucaramanga; Observatorio de Salud Publica de Santander. 2018.

3. World Health Organization.OMS. Injuries Violence the Facts. 2010. https://apps.who.int/iris/bitstream/ handle/10665/44288/9789241599375_eng. pdf? sequence $=1$
4. World Health Organization. Global status report on road safety 2018. https:/issuu.com/globalncap/ docs/who-status-report-eng

5. Departamento Administrativo Nacional de Estadisticas 2018. Estadisticas Vitales - EVV cifras definitivas 2017. https://www.dane.gov.co/files/ investigaciones/poblacion/cifras-definitivas-2017. pdf

6. Gómez H, Castro VM, Franco-Marina F, Bedregal M, Rodriguez J. et al. La carga de la enfermedad en países de América Latina. Salud Publica Mex. 2011; 53(2): 572-577.

7. Montero OY, Ayala CM, Esquiaqui FR. Estudio de morbilidad por causa externa, Bucaramanga. 2016; Aplicatec. 2018; 8(2): 1-5.

8. Murray C, Lopez AD. The global burden of disease. Global burden of disease and injury series. Vol I. World Health Organization; 1996.

9. Departamento Administrativo Nacional de Estadística DANE. (n.d.). Estadísticas vitales nacimientos y defunciones. http://www.dane.gov.co/ index.php/estadisticas-por-tema/salud/nacimientosy-defunciones

10. Murray C, López A. The global burden of disease. Global burden of disease and injury series. Vol I. Cambridge Harvard School of Public Health. Harvard University Press. 1996; p. 412-416.

11. Murray C, López A. Global health statistics. Global burden of disease and injury series. Vol II. Cambridge: Harvard School of Public Health. Harvard University Press. 1996.

12. Dismod II. Programa desarrollado por la Organización Mundial de la Salud. Versión 1.04. University of Queensland: School of Population Health 2001-2008.

13. Castaneda-Porras A, Segura O. Carga de enfermedad por lesiones de causa externa en Casanare, Colombia, 2008-2012. Rev Salud Pública. 2018; 20(2): 163-170. 